\title{
Audit (lower GI tract) F265-F271
}

F265

ABDOMINAL ULTRASOUND IN THE DETECTION OF COLONIC LESIONS:

D Kumar, A Joseph*. Department of Colorectal Surgery \& Radiology*, St George's Hospital, London, UK.

Colonic pathology is conventionally investigated by barium enema (BE) or colonoscopy. These are invasive investigations and require considerable pre-investigation preparation. The aim of this study was to investigate the efficacy of abdominal ultrasound (US) in identifying colonic lesions.

We have studied 33 consecutive patients who were suspected of having colorectal cancer. The gold standard was either a barium enema or colonoscopy with biopsy. All patients had an abdominal ultrasound performed by the same consultant radiologist who was blinded to the result of the BE or colonoscopy.

Eight patients had an abdominal ultrasound before the BE or colonoscopy. A colonic lesion was identified on US in $32 / 33$ (97\%) patients. In $29 / 33$ (88\%) this was correct for the site and nature of the lesion. In two patients, although the pathology was identified correctly, the site was diagnosed incorrectly. In another patient, who had advanced caecal carcinoma with peritoneal spread, a diagnosis of ileocaecal tuberculosis was made on US. In one patient with tumour in the descending colon on $\mathrm{BE}$, the lesion was missed on US. In the 8 patients who had the US first, the diagnosis of colonic cancer (associated colovescical fistula in 2) was made correctly in all. In 2 patients the diagnosis was missed on BE but correctly spotted on US.

These data show that it is possible to detect colonic cancer on abdominal ultrasound. It is not a substitute for $\mathrm{BE}$ or colonoscopy but may be useful in the elderly or a difficult patient. A bigger controlled study is required to validate this further.
F267

CLOSURE OF LOOP ILEOSTOMY

DP Berry and J H Scholefield

Univ. Lept. of Surgery, E Floor, University Hospital, Nottingham.

Introduction: The formation of a proximal definctioning stoma for low pelvic anastomoses is considered standard practice by meny. Data from prospective, randomised trials has demonstrated that loop ileostomy is preferable to loop colostomy because it is less likely to prolapse or herniate, the effluent has less odour and it can be accurately sited preoperatively for greater patient comfort than loop colostomy. Despite these advantages, the major reason surgeons in our institution cited for not using loop ileostomy is that closure is difficult.

Aim: The aim of this study was to audit our results for the closure of loop ileostomy using a totally stapled technique.

Method: 16 consecutive patients (13 men, 3 women, median age 62.5

yrs, range 22-75 yrs) were identified. Indications for the stoma were 13 low anterior resections, 3 ileoanal pouches and 1 reveral of Hartmanns procedure. Stomas were closed by side-to-side stapling with a TLC-75 (Ethicon, UK) inserted into the two limbs and the loop subsequently closed by cross stapling the apex.

Results: Before closure, 1 patient developed a parastomal hernia. The stomas were closed after a medim interval of 14 weeks. (range 2-32 weeks) and took a median operative time (skin incision to skin closure) of 32.5 minutes (range 15-55 minutes). Following stoma closure gastrointestinal function returned (flatus passed) after a modim interval of 3 days (range 2-5 days) and patients were hospitalised for 6 days (range 4-8 days). One patient suffered a wound infection. No patient suffered anastomotic leakage or small bowel obstruction.

Conclusion: Closure of loop ileostomy by this stapled technique is simple, quick, effective and safe. We advocate loop ileostomy as the defunctioning stoma of choice.
ENDOANAL ULTRASONOGRAPHY AND ANORECTAL PHYSIOLOGY IN PATIENTS WITH FAECAL INCONTINENCE. Benson $\mathrm{M}^{*}$, Kumar $\mathrm{D}$, Jazarwi $\mathrm{R}^{*}$, Lloyd $\mathrm{R}^{*}$, Leicester R. Grant E. Departments of Colorectal Surgery and Gastroenterology*. St George's Hospital, Tooting, London, UK.

The aetiology of faecal incontinence is multifactorial. Importan causes include direct trauma to the sphincter during childbirth, pudendal neuropathy and abnormalities of rectal compliance. The aim of this study was to assess the diagnostic efficacy of endoanal ultrasonography and anorectal physiology in patients with faecal incontinence.

We studied 118 patients with a mean age of 51.2(1.9) years. All patients had Grade III to Grade IV incontinence. Endoanal ultrasonography revealed sphincter defects in 66 patients (internal anal sphincter (IAS) 8, external anal sphincter (EAS) 30 and IAS and EAS 28). 52 patients had no demonstrable sphincter defects on ultrasonography. Anal manometry showed that in the IAS defect group, 5 patients had low basal pressures and 2 patients had low squeeze pressures as well. In the EAS defect group 21 patients had low basal pressures and a total of 23 patients had low squeeze pressures. In the combined defect group 6 patients had normal pressures. 19 had low basal pressures, 13 had low squeeze pressures. In the 52 patients with no defects only 29 had either low squeeze pressures or low basal pressures. The squeeze and basal pressures were not independent variables but showed a strong correlation for the total group $(r=68, P<0.0001)$. In the 27 patients who had no sphincter defects and had normal pressures, rectal sensation revealed impairment of constant volume sensation and maximum tolerable volume sensation in 20 and of constant volume sensation alone in 7 . None of the patients had impairment of maximum tolerable volume alone. These data show that a single test alone will only diagnose the underlying problem in approximately $50 \%$ of patients whereas a combination of endoanal ultrasonography, anorectal physiology and rectal volume sensation will provide the answer in almost every patient.
10 YEARS' EXPERIENCE IN TREATING METASTATIC NEUROENDOCRINE TUMOURS. AJ Stangou', R Sherwood ${ }^{2}, H$ Nunnerly ${ }^{3}$, B Portmann', J Karani ${ }^{3}$, E Howard, I Benjamin ${ }^{4}$, J Ramage', Roger Williams'. Institute of Liver Studies', Depts of Biochemistry ${ }^{2}, \quad$ Radiology ${ }^{3}$, and Hepatobiliary Surgery", King's College Hospital, London SES 9RS, U.K.

Introduction. Neuroendocrine tumours usually run a slow. course even in the presence of metastatic disease at diagnosis, with many therapeutic options for surgical or medical management. This study is a review of 79 cases of metastatic neuroendocrine tumours treated in our centre over a 10 year period. Patients and methods. 43 male and 36 female patients aged 29-78 years were referred with metastatic liver lesions, or symptoms of flushing (64\%), diarrhoea ( $44 \%$ ), abdominal pain (6\%), jaundice $(5 \%)$, breathlessness $(8.7 \%)$, oedema (6\%), of 1 month-37 years duration. 67 patients were diagnosed on the basis of positive 5 HIAA screen ( $>50 \mu \mathrm{mol} / 24$ hours), imaging, or histology, and 12 at laparotomy. 18 patients had resection of the primary tumour and 9 patients received orthotopic liver transplantation. Of 54 patients with carcinoid syndrome 39 were treated with 1-6 sessions of embolisation and 12 with octreotide. 14 patients were treated with 5-fluorouracil (5-FU) and streptozotocin( STZ). Results. All 79 patients had liver metastases, and 7 lymph node involvement. Resection of primary in 18 cases was associated with survival of 1-38 years. Of the 9 transplanted patients 3 survived over 4 years. Both embolisation and octreotide treatment resulted in resolution of symptoms and 55\% decrease in the 5-HIAA level in $85 \%$ and $80 \%$ of cases respectively; steatorrhoea in 4 and cholelithiasis in 1 cases was seen with octreotide. Objective response was seen with the 5-FU and STZ regimen and in combination with embolisation exceeded 5 years in over $50 \%$ of cases; main side effect, nephrotoxicity. Combination of surgical treatment with embolisation or/and chemotherapy resulted in a median survival of 9.7 years. Conclusions Octreotide has similar effect to hepatic artery embolisation in symptomatic relief and hormone levels. Combination treatment of surgical resection/embolisation/ chemotherapy appears to offer the best results. Chemotherapy with 5FU and STZ has a role in advanced disease, but renal function needs to be closely monitored. 
CLINICAL ASSOCIATIONS WITH BACTERIAL

TRANSLOCATION

O'Boyle CJ, Sagar PM, Murchan P, Mitchell CJ, MacFieJ

Combined Gastroenterology Service, Scarborough

Hospital, North Yorkshire

Gut translocation of bacteria (BT) may be important in the aetiology of multi-organ failure. Surprisingly little information is available as to the prevalence of this phenomenon in different patient groups. In this study gut translocation of bacteria was assessed by culture of mesenteric lymph nodes and serosal scrapings taken at laparotomy. Standard microbiological techniques were used.

A total of 448 patients was studied BT to mesenteric lymph nodes was identified in 69 patients (15.4\%). Comparison of individual patient groups revealed no significant differences in the incidence of translocation between benign and malignant disease ( $17 \%$ versus $13 \%$ ), inflammatory bowel disease and other GI disease ( $20 \%$ versus $15 \%$ ), significant weight loss and no weight loss (10\% versus $15 \%)$ and total parenteral nutrition and enteral feeding ( $18 \%$ versus $14 \%)$. However,

translocation was more common in patients with intestinal obstruction $(30 \%)$ than in those without obstruction $(13 \%, p<0.01)$. The more distal the level of obstruction, the greater the incidence of translocation. Postoperative septic complications were more common in patients with confirmed BT $(p<0.001)$

We conclude that the incidence of BT is highest in patients with intestinal obstruction.

F270

BACTERIAL COLONIZATION OF THE UPPER GASTROINTESTINAL TRACT IS ASSOCIATED WITH SEPTIC MORBIDITY IN SURGICAL PATIENTS

OBoyle CJ, Murchan P, Dave K, Mitchell CJ, MacFie J.

Combined Gastroenterology Unit, Scarborough General Hospital,

Scarborough, North Yorkshire.

Bacterial colonization of the normally sterile upper gastrointestinal tract may predispose to septic morbidity in surgical patients. We have attempted to confirm this in a prospective study in which changes in proximal gut flora and septic complications have been recorded.

All patients underwent nasogastric intubation as part of standard c̊llnical treatment. Gastric aspirate $(10 \mathrm{ml})$ was obtained under sterile conditions and sent for microbiological culture. All septic events were recorded and the microbiology assessed.

A total of 231 patients were studied. Sixty five patients (28\%) had sterile cultures, seventy two $(31 \%)$ grew one organism type and $95(41 \%)$ had multiple organisms cultured from their aspirates. Monoand multiorganism culture were associated with a significant increase in septic complications when compared with no growth (38\% vs $17 \%$ $\left.p<0.05 x^{2}=4.16\right)$ and $\left(56 \%\right.$ vs $\left.17 \%, p<0.001 x^{2}=11.52\right)$ respectively. Coliforms and particularly E. Coli and Strep. Faecaelis were strongly associated with subsequent septic events $(68 \% p<$ $0.001,87 \% \mathrm{p}<0.001$ )

We conclude that colonisation of the upper gastrointestinal tract frequently occurs in ill surgical patients and does predict septic morbidity.
SEQUELAE OF INFECTIOUS DIARRHOEA: HIGH RATE OF GASTRO-INTESTINAL INVESTIGATIONS AND LEVELS OF MORBIDITY. J.M. Hebden ${ }^{1}$, RC. Spiller ${ }^{1}$, K.R Neal ${ }^{2}$. Depts. of Gastroenterology ${ }^{1}$ and Epidemiology ${ }^{2}$, University of Nottingham Medical School, UK.

The number of cases of notified food poisonings continues to increase. However, little is known about the consequences of food poisoning in terms of patient morbidity and medical care and resource usage.

The statutory notifications system was used to identify 665 people with gastroenteritis over a 6 month period. A questionnaire covering medical care, the episode of gastro-enteritis and sequelae, bowel habit now and 12 months before was mailed 6 months after the notification date. 435 questionnaires (66\%) were retumed. Statistical analyses were done using SPSS for windows.

49 patients $(11 \%)$ were admitted to hospital with no difference in admission rates by age, sex or pathogen. There was 1 death and 1 patient with a perforated colon leading to admission to intensive care. $168(39 \%)$ of the patients had at least 7 days off from work or regular duties. This is an underestimate as very few people above retirement age reported time off work. 90 patients reported weight loss of at least 1 stone with a median time of 8 weeks before regaining the weight, 16 had not regained their original weight at 6 months. Significant weight loss was associated with male sex relative risk $(R R)=1.7(95 \% \mathrm{CI}=1.1-2.6)$, duration of diarthoea $R R=1.5(95 \% \mathrm{CI}=1.2-1.8)$ for each week of symptoms and vomiting as part of the illness $R R=1.5(95 \% C I=1.1-2.1)$, after controlling for hospital admiscion. 107 patients (25\%) reported a persistent change in bowel habit at 6 months, of which $28(7 \%)$ fitted the Rome criteria for IBS. 21 patients $(5 \%)$ had further investigations including 5 barium enemas, 1 barium meal, 2 OGDs. with the remainder having out-patient assessments.

In conchusion gastro-enteritis results in significant morbidity, time off work and health care resource utilisation.

\section{'Non-steroidals' F272-F277}

F272

MESENTERIC MARGINAL ULCERATION: PREDILICTION FOR CRITICALLY PERFUSED AREAS IN INDOMETHACININDUCED JEJUNAL ULCERS IN THE RAT. A Anthony, C Thrasivoulou, RE Pounder, AJ Wakefield, AP Dhillon Inflammaton Bowel Disease Study Group, Royal Free Hospital School of Medicine, London, UK.

Background/Aim; This study tested the hypothesis that mesenteric marginal ulceration, a feature of both Crohn's disease and indomethacin(indo)-induced villous contraction and subsequent jejunal ulceration in the rat, localises to critically.perfused areas of the gut. Two separate sets of experiments were performed in the rat to $(a)$, correlate the site of indo-induced intestinal injury with the normal vascular anatomy and (b), to compare regional susceptibility of the intestine to injury following either indo or a general insult, that is, ischaemia-reperfusion following mesenteric artery occlusion. Methods; Rats ( $\mathrm{n}=5 /$ group) received either $(a)$ oral indo $10 \mathrm{mg} / \mathrm{kg}$ or vehicle and terminated $4 \mathrm{~h}$ later. The mesenteric artery was perfused with carbon ink and the intestines formalin-fixed. Tissues were cleared with methyl salicylate for visualisation of the vasculature by dissection and light microscopy. (b) Alternatively. anaesthetised rats ( $n=5 /$ group) were treated as follows; bolus indo iv $75 \mathrm{mg} / \mathrm{kg}$ or iv vehicle with histological examination of the intestines $20 \mathrm{~min}$ later; mesenteric artery occlusion $(10 \mathrm{~min})$ then reperfusion (10 min); sham operation $20 \mathrm{~min}$. Results; (a) All indodosed rats, but none of the controls, developed visible focal areas of severe villous contraction at $4 \mathrm{~h}, 99 \%$ of which were localised between vasa recta along the mesenteric margin. Arterial imaging of the control rat small intestines identified a paucity of the submucosal plexus between vasa recta adjacent to jejunal, but not duodenal or ileal, mesenteric margins; in contrast, the jejunal submucosal plexus was extensive on the anti-mesenteric side. (b) Indo $75 \mathrm{mg} / \mathrm{kg}$ iv caused villous contraction of jejunal, but not duodenal or ileal, mesenteric villi between vasa recta. Mesenteric artery ischaemiareperfusion caused shortening of all villi in the jejunum/ ileum. The villi of sham controls were normal. Conclusions; Critically perfused areas of the mesenteric margin of the rat jejunum are susceptible to NSAID injury. Indo-induced villous contraction that precedes jejunal ulceration may arise as a consequence of ischaemia/reperfusion injury to critically perfused areas of the jejunum.

(This study was supported by Glaxo Wellcome, UK). 